Published in "Journal of Colloid and Interface Science 562(): 502-510, 2020"

which should be cited to refer to this work.

\title{
Understanding the assembly of amphiphilic additives in bulk and dispersed non-lamellar lipid-based matrices: Phosphorylation, H-bonding and ionisation
}

\author{
Marco F. Etter ${ }^{\mathrm{a}}$, Daria Dellenbach ${ }^{\mathrm{a}}$, Alke Petri-Fink ${ }^{\mathrm{b}}$, Barbara Rothen-Rutishauser ${ }^{\mathrm{b}}$, Ehud M. Landau ${ }^{\mathrm{a}, *}$, \\ Wye-Khay Fong b,1,*
}

${ }^{a}$ Department of Chemistry, University of Zürich, Winterthurerstrasse 190, 8057 Zürich, Switzerland

${ }^{\mathrm{b}}$ Adolphe Merkle Institute, University of Fribourg, Chemin des Verdiers 4, 1700 Fribourg, Switzerland

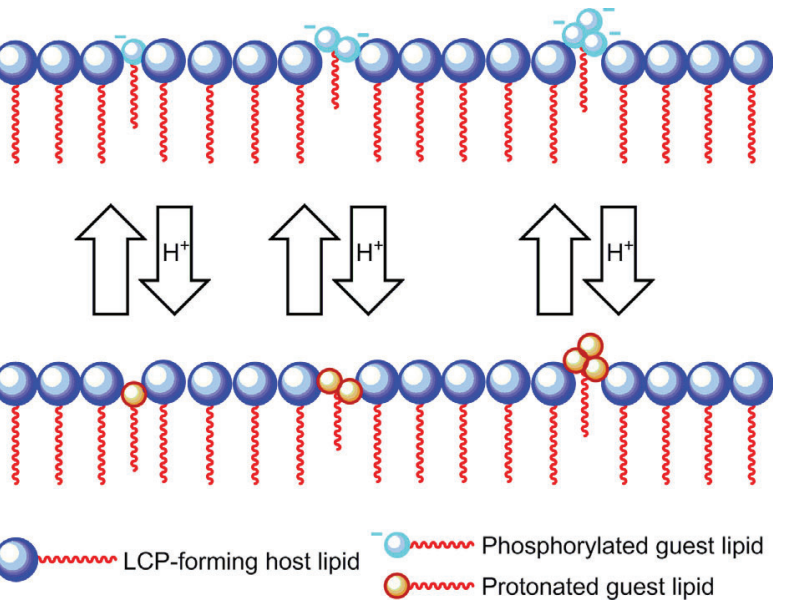

A R T I C L E I N F O

\section{Article history:}

Received 7 August 2019

Revised 19 November 2019

Accepted 19 November 2019

Available online 21 November 2019

\begin{abstract}
A B S T R A C T
The aqueous channel size of lipidic cubic phases can be a limiting factor for certain applications. For this reason, additives have been used to exquisitely control their nanostructure. In this study, two families of primary phosphoesters have been designed, synthesised and utilised to determine the effect of the positioning of the guest additive at the interface of the host mesophase, and to contrast the effect of headgroup ionisation and protonation. A general methodology has been developed to produce primary phosphoesters, and a unique use of ${ }^{31} \mathrm{P}$ NMR has been used in order to systematically investigate the influence of these additives on monoolein- and phytantriol-based bulk lipidic cubic phases and dispersed cubosomes. In general, di-phosphorylated additives exhibit a greater effect upon lipid packing than the mono- and tri-phosphorylated molecules due to their optimal positioning. In dispersion, the protonation state of the phosphate headgroups was manipulated by altering the $\mathrm{pH}$, where shifts in $\mathrm{pK}_{\mathrm{a}}$ determined
\end{abstract}

\footnotetext{
* Corresponding authors.

E-mail addresses: ehud.landau@chem.uzh.ch (E.M. Landau), khay.fong@newcastle.edu.au (W.-K. Fong).

1 WKF: Chemistry, School of Environmental \& Life Sciences, The University of Newcastle, Callaghan, NSW, 2308 Australia.
} 
by ${ }^{31} \mathrm{P}$ NMR were used as a fluorescent label-free method to identify the location and ionisation state of the phosphate additives. This study systematically evaluates the influence of the positioning of the additive, headgroup size and charge of phosphorylated lipids on the behaviour of lipidic mesophases.

\section{Introduction}

Lipidic cubic phases (LCPs) are nanomaterials that have potential for utilisation in various applications such as catalysis, drug delivery and membrane protein crystallisation or reconstitution [1-6]. These applications are dependent on intermolecular interactions at the lipid-water interface. The maximal diameter of the aqueous channels in LCPs composed of monoolein (MO) and water is around $40 \AA$, and can be a limiting factor [7]. There is ongoing research on the effect of additives on the lipid-water systems that form LCPs in order to control the size of the aqueous channels and phase transitions. A plethora of hydrophobic and amphiphilic guest molecules have been introduced into host liquid crystalline matrices, where the influence of additives on the swelling and phase transitions of lipidic mesophases is determined by the impact of the additives on the curvature of the bilayer [2,7-17]. For LCPs, and inverted liquid crystalline mesophases in general, the truncated conical shape of the lipid molecule results in the formation of negatively curved structures. The addition of amphiphilic guest molecules that have a larger head to tail group ratio forces a decrease in the overall curvature of the lipidic membrane. This has been demonstrated with amphiphilic additives, such as sugar surfactants and phospholipids, which preferentially affect the headgroup region $[2,9,13,15]$. In contrast, hydrophobic additives such as cholesterol, vitamin E acetate and oleic acid, influence the lipidic portion of the cubic nanostructure, thereby increasing the overall curvature of the membrane $[11,18,19]$. As can be seen, the molecular features of the additives that change the ratio of headgroup to hydrophobic tail volume, can dramatically influence the packing of lipids in the cubic phase.

In addition to the molecular features of the additives, intermolecular phenomena play a major role in affecting headgroup interactions, specifically: hydrogen bonding, where headgroups can form a network of interactions with adjoining headgroups and bound water; and hydration, where the amount of water coordinated around the headgroup affects the surface interaction of molecules at the interface $[8,20-22]$.

Lipids that contain multiple charges are particularly adept at manipulating lipid curvature. One of the most commonly utilised have been phospholipids, which usually have a single charge or are zwitterionic $[8,11,13,23,24]$. Minimal amounts of these ubiquitous charged phospholipids can introduce a phase transition and drastically increase the lattice parameter of LCPs [11,25]. However, it is unclear from these studies which component of the additive has the greatest influence upon the membrane curvature: the functionalisation of the headgroup or the ionisation state of the headgroup.

In this study, a series of primary phosphorylated lipids have been designed and synthesised, and their influence on the lipidic cubic mesophase investigated. The phosphorylated lipids possess a single lipidic tail to anchor the molecule to the lipid-water interface, and a headgroup containing between one and three phosphates. The lipidic tails comprise of either a relatively short $C_{12}$ chain or a more complex phytol-based chain, chosen based upon previous studies which suggested that amphiphiles with these tails have the greatest influence on a cubic phase bilayer [26]. With this small library of phosphate surfactants, this study aims to understand the effect of the molecular properties of the headgroup of the additives on the ensuing lipidic mesophase by investigating the effect of the number of primary phosphates attached to the headgroup as well as the ionisation state of the headgroup. Thus, this report provides a systematic study into the effects of headgroup size and protonation state upon the bilayer curvature in a monoacylglycerol- or phytantriol-based cubic phase.

\section{Materials \& methods}

\subsection{General information related to synthesis and pH-measurements}

All chemicals were used as received unless otherwise stated. All solvents were of technical grade and distilled prior to their use. Solvents were removed from the products in vacuo at standard bath temperatures and pressures [27]. Column chromatography was performed using silica gel Merck 60 (particle size 40-63 $\mu \mathrm{m}$ ) or Q-Sepharose ${ }^{\circledR}$ Fast Flow (preswollen, 45-165 $\mu \mathrm{m}$ ) (SigmaAldrich) with the indicated solvent system. Analytical thin-layer chromatography (TLC) was performed using Merck pre-coated silica gel plates $60 \mathrm{~F} 254$, visualisation was performed by UV light (254 nm and $366 \mathrm{~nm}$ ) and stains if indicated. Medium Pressure Liquid Chromatography (MPLC) was performed by CombiFlash ${ }^{\circledR}$ $\mathrm{Rf}+$ Lumen $^{\mathrm{TM}}$ (TELEDYNE ISCO) with indicated column size, solvents, time and flow rate. Freeze-drying of aqueous samples was performed at a CHRIST alpha 1-4 LD plus after freezing the samples in liquid nitrogen. ${ }^{1} \mathrm{H}$ NMR, ${ }^{13} \mathrm{C}$ NMR, ${ }^{31} \mathrm{P}$ NMR and ${ }^{31} \mathrm{P}\left[{ }^{1} \mathrm{H}\right]-\mathrm{NMR}$ spectra were recorded on the following machines: Bruker AV-300 (300 MHz), Bruker AV-400 (400 MHz) or Bruker AV-500 $(500 \mathrm{MHz})$. Chemical shifts are given in parts per million ( $\mathrm{ppm}$ ) relative to the internal standard. Representative spectra can be found in the SI. Coupling constants, J, are expressed in $\mathrm{Hz}$ and multiplicities are abbreviated as follows: $\mathrm{s}$ (singlet), br (broad), $\mathrm{d}$ (doublet), $\mathrm{t}$ (triplet), q (quadruplet), quint (quintet), $\mathrm{m}$ (multiplet). Peaks in ${ }^{13} \mathrm{C}$ NMR that were not completely separated are named $2 \mathrm{p}$ for two peaks and $\mathrm{mp}$ for more. Mass spectra were recorded by the Mass Spectroscopy Service of UZH on Finnigan MAT95 MS, Bruker LC MS and Finnigan TSQ700 MS machines.

For all synthesised molecules, phosphorylation was followed by the deprotection of the phosphate-protecting group resulting in the final product (Fig. 1).

\subsection{Formation of bulk and dispersed cubic phases (Cubosomes)}

For the bulk studies, monoolein (MO, >99\%) was purchased from NU-CHEK Prep. (MN, USA) and phytantriol (PHYT) was sourced from DSM (Netherlands). All measurements were performed in excess of water. For the dispersed samples Dimodan, a commercial product containing $>98 \mathrm{wt} \%$ monoacylglycerols (the major component being monolinolein, MLO) was used as received from Danisco. The same batch of Dimodan was used throughout the work. Pluronic F127 was purchased from Sigma Aldrich. The phosphorylated lipids, $\mathrm{C} 12 \mathrm{P} 1$ and $\mathrm{C} 12 \mathrm{P} 2$, were mixed at predetermined concentrations into the bulk lipid by three cycles of heating to $60{ }^{\circ} \mathrm{C}$ and vortexing until becoming homogeneously viscous. Homogenisation of C12P3, PhyP2 and PhyP3 into bulk lipids (MO, MLO and PHYT) required dissolving the pre-weighed mixtures in methanol containing $0.001 \%$ formic acid and subsequent removal of the solvent under vacuum at $50{ }^{\circ} \mathrm{C}$ for $>24 \mathrm{~h}$. 

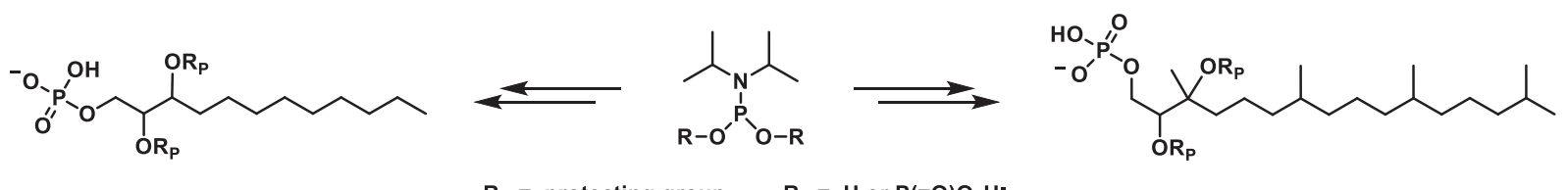

Fig. 1. Schematic overview of the lipid syntheses. Left: Phosphorylation and deprotection of the $\mathrm{C}_{12}$-chain; Right: Phosphorylation and deprotection of the phytol chain. The full synthetic route can be found in the Supporting Information.

For the bulk phases, the lipids were subsequently hydrated in Tris buffer ( $50 \mathrm{~mm}, \mathrm{pH} 6$ or 8 ) or citric acid buffer $(50 \mathrm{~mm}, \mathrm{pH} 3)$. The lipidic and aqueous components were thoroughly mixed by passing the mixture through a coupled syringe mixing device (Formulatrix, MA, USA), between two $100 \mu$ gas-tight syringes (Hamilton CA, USA). Lipid dispersions were formed by hydrating lipids in a solution of Pluronic F127 (0.5 wt\% F127, 5 wt\% lipid, pH 7.4) before being dispersed by probe ultrasonication ( 3 min pulsed, $30 \%$ amplitude) to reduce and homogenise the particle size. The $\mathrm{pH}$ of the samples was adjusted by addition of appropriate amounts of $\mathrm{HNO}_{3}(0.1$ or $0.001 \mathrm{~m})$ and $\mathrm{KOH}(0.1$ or $0.001 \mathrm{~m})$. Samples were left to equilibrate for $\geq 12 \mathrm{~h}$.

\subsection{Small angle X-ray scattering (SAXS) measurements}

SAXS measurements were carried out on a NanoMAX-IQ instrument (Rigaku) with a Pilatus $100 \mathrm{~K}$ detector (Dectris), a Cu target sealed tube source (MicroMax-003, Rigaku) in vacuum and at controlled temperature (Julabo). An effective scattering-vector range of $0.008 \AA^{-1}<q<0.6 \AA^{-1}$ was investigated and scattering images were integrated into the one-dimensional scattering function $I(q)$. The scattering intensity is shown as a function of the momentum transfer $q=4 \pi \lambda^{-1} \sin (\theta / 2)$, where $\lambda$ is the photon wavelength of $1.524 \AA$ and $\theta$ is the scattering angle. Samples were filled into a $1.5 \mathrm{~mm}$ internal diameter quartz capillary and sealed with epoxy glue. The $q$-range was calibrated using silver behenate as the standard. The liquid crystalline space groups and lattice parameters $(a)$ were determined by the relative positions of the Bragg peaks in the scattering curves, which correspond to the reflections on planes defined by their $(h k l)$ Miller indices [28].

\subsection{Dynamic light scattering}

Particle size were obtained by dynamic light scattering (DLS) measurements (Brookhaven 90 Plus Particle Size Analyzer, Brookhaven, Holtsville, USA). Briefly, dilute solutions of the as-made lipid particles were placed into a plastic cuvette; each had a $\mathrm{pH}$ of $\sim 6$. Each analysis to obtain the z-average and polydispersity index (PDI) was based on a repetition of eight measurements.

\section{5. $p K_{a}$ measurements - Titration and ${ }^{31} P$ nuclear magnetic resonance (NMR) spectroscopy}

The synthesised phosphorylated lipids were sparingly soluble in water, except for C12P1, which was insoluble except in basic conditions at $\mathrm{pH}>8$. Phosphorylated lipids $(0.01 \mathrm{~mm})$ were dissolved in $\mathrm{H}_{2} \mathrm{O}(0.4 \mathrm{~mL})$ and $\mathrm{D}_{2} \mathrm{O}(0.05 \mathrm{~mL})$ in Eppendorf tubes. Lipid dispersions were diluted with $10 \% \mathrm{D}_{2} \mathrm{O}$ for NMR analysis. The $\mathrm{pH}$ of the samples was adjusted with solutions of $\mathrm{NaOH}(0.25 \mathrm{~m}$ to $1 \mathrm{~m})$ and $\mathrm{HCl}(0.25 \mathrm{~m}$ to $1 \mathrm{~m})$ and measured with a $\mathrm{pH}$ meter (CyberScan 500 , microelectrode). The measured NMR spectra showed a clear pH dependence, enabling titration curves to be plotted. Representative spectra can be found in the SI. Spectra were processed with Mestrenova and subsequently plotted with Origin 8.0, using a recently published fitting Eq. (1) [29].
$P_{\text {total }}=\frac{\left(P_{A^{2-}}\right)+\left(P_{A H^{-}}\right) 10^{n\left(p K_{a 2}-p H\right)}}{1+10^{n\left(p K_{a 2}-p H\right)}}$

The used parameters are: $P_{A^{2-}}$, the chemical shift of the deprotonated phosphate; $P_{A H^{-}}$, the chemical shift of the singly protonated phosphate; and $n$, the slope of the curve.

\section{Results and discussion}

\subsection{Synthesis of phosphorylated lipids}

Synthesis of three phosphorylated lipids is depicted in Scheme 1, in which commercially available precursors are phosphorylated with subsequent deprotection. Purification of the synthesised multiphosphorylated lipids (6 (C12P2) and 9 (PhyP3)) was achieved by ion exchange chromatography using QSepharose ${ }^{\circledR}$ columns, resulting in the target lipids with yields of around $90 \%$.

In Scheme 2 the three-step synthesis of the two additional phosphorylated lipids is depicted. To prepare the two desired starting materials, a SHARPLESS dihydroxylation was required on the pathway to 13 (C12P3) and in the case of 17 (PhyP2), a hydroxyl group was introduced via hydroboration at the double bond of $\mathbf{1 4}$.

\subsection{Mesophases containing phosphorylated lipids}

\subsubsection{Phase behaviour of bulk LCP with phosphorylated lipidic additives}

In order to establish the effects of the guest synthetic phosphorylated lipids on the host lipidic cubic phases, they were incorporated into both monoolein (MO)- and phytantriol (PHYT)-based bulk and dispersed mesophases. Initially, bulk systems were investigated to determine the effect of different concentrations and heating behaviour of the formed mesophase.

3.2.1.1. Phase behaviour of MO bulk phase with $\mathrm{C} 12$ and phytol phosphorylated lipids. The effect of incorporating various phosphorylated additives into MO mesophases was investigated via SAXS (Fig. 2A-F, changes in lattice parameters and raw SAXS data are reported in the Supplementary Information, Fig. SI1-5 and Fig. SI9). In the temperature and hydration range investigated, MO displays a double diamond bicontinuous cubic symmetry $\left(\mathrm{V}_{2}\right.$ Pn3m). The lipids with the lowest number of phosphate groups per molecule (C12P1 and PhyP2) influence the phase behaviour in a way similar to that of typical hydrophobic additives. In the case of PhyP2, both an increase in additive concentration and temperature leads to the formation of inverted hexagonal phase $\left(\mathrm{H}_{2}\right)$ indicating that the C12P1 and PhyP2 additives are swelling the hydrophobic volume of the mesophase. Interestingly, increasing the temperature of $0.5 \mathrm{~mol} \% \mathrm{C} 12 \mathrm{P} 1$ at $\mathrm{pH} 3$ avoids the formation of the $\mathrm{H}_{2}$ phase and the formation of inverse micelles $\left(L_{2}\right)$ was observed. Upon increasing the number of phosphates per molecule an opposite behaviour is observed: the C12P2, C12P3 and PhyP3 additives promote swelling of the $\mathrm{V}_{2} \mathrm{Pn} 3 \mathrm{~m}$ unit cells, whereby this effect is $\mathrm{pH}$ dependent, and is greatest at $\mathrm{pH} 3>6>8$, and transi- 


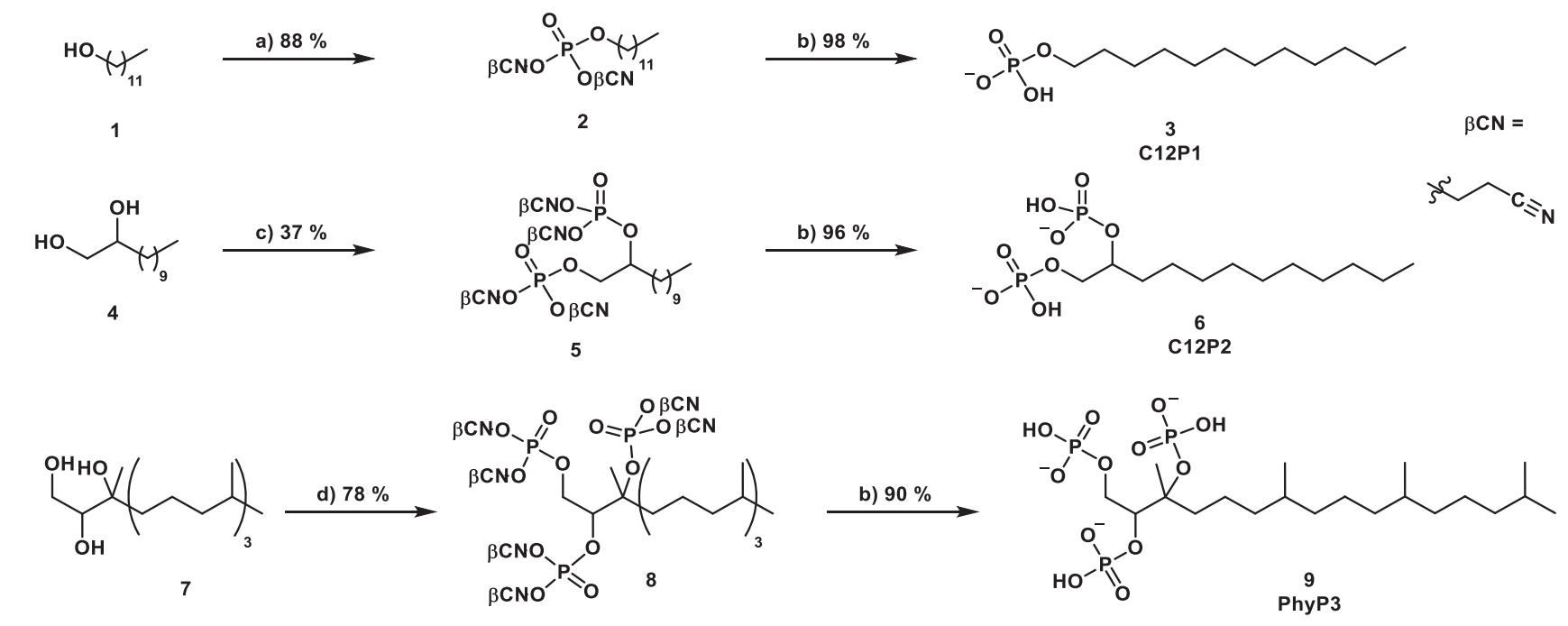

Scheme 1. Synthesis of phosphorylated lipids. Reagents and conditions: (a) i. bis(2-cyanoethyl)diisopropylphosphoramidite, tetrazole, $\mathrm{MeCN}$, r.t., 4 h; ii. $t \mathrm{BuOOH}, 0{ }^{\circ} \mathrm{C}$, $20 \mathrm{~min}$; (b) $25 \% \mathrm{NH}_{4} \mathrm{OH} / \mathrm{H}_{2} \mathrm{O}, \mathrm{MeOH}, 60^{\circ} \mathrm{C}$, o.n.; (c) i. bis(2-cyanoethyl)diisopropylphosphoramidite, 5-(phenyl) $1 H$-tetrazole, DMF, r.t., 6 h.; ii. $t$ BuOOH, $0{ }^{\circ} \mathrm{C}, 20$ min; (d) bis(2cyanoethyl)diisopropylphosphoramidite, 5 -(phenyl) $1 \mathrm{H}$-tetrazole, DMF, r.t., 6 h; ii. $t \mathrm{BuOOH}$, decane, $0{ }^{\circ} \mathrm{C}, 20 \mathrm{~min}$.<smiles>F[AsH]CC1c2ccccc2-c2ccccc21</smiles>

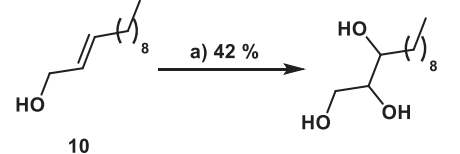<smiles>C1C[13CH]2C3CC2C13</smiles>

11

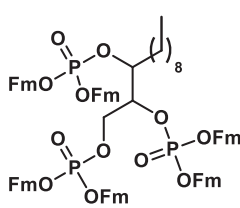

12

\section{c) $90 \%$}

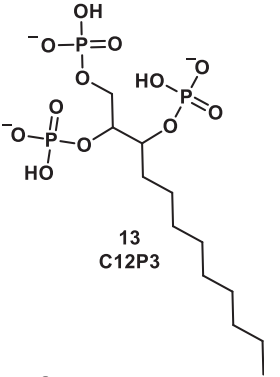<smiles>CC(=CCO)CCCC(C)C</smiles><smiles>CC(C)CCCC(C)C(O)CO</smiles>

15

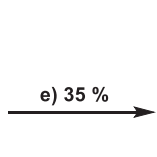

$\beta \mathrm{C}$

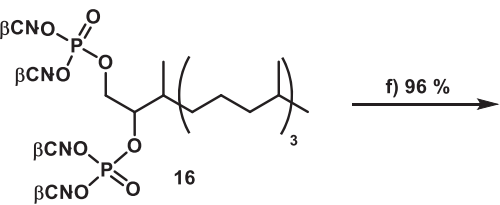

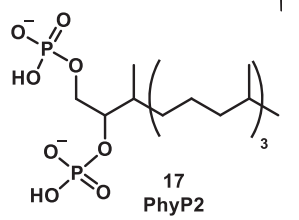

Scheme 2. Reaction pathways towards the additional phosphorylated lipids to complete the library. Reagents and conditions: (a) i. MD-mix- $\alpha$, $i$ PrOH, $\mathrm{H}_{2} \mathrm{O}, 0{ }^{\circ} \mathrm{C}$ to r.t., $0 . n$.; ii) $\mathrm{NaSO}_{3}, 0^{\circ} \mathrm{C}, 1$ h; (b) i. bis(Fm)diisopropylphosphoramidite, tetrazole, THF, r.t., 6 h; ii. $t \mathrm{BuOOH}, 0{ }^{\circ} \mathrm{C}, 20$ min; (c) Piperidine, $\mathrm{CH}_{2} \mathrm{Cl}_{2}$, r.t., 2.5 h; (d) i. Me $2 \mathrm{~S}$ BH3, THF, r.t., 4 h; ii. $\mathrm{NaOH}, \mathrm{H}_{2} \mathrm{O}_{2}, \mathrm{H}_{2} \mathrm{O}, 0^{\circ} \mathrm{C}, 30 \mathrm{~min}$; (e) i. bis(2-cyanoethyl)diisopropylphosphoramidite, 5-(phenyl) $1 \mathrm{H}$-tetrazole, $\mathrm{DMF}, \mathrm{r.t}$., 4 h.; ii. $t \mathrm{BuOOH}, 0{ }^{\circ} \mathrm{C}, 20 \mathrm{~min}$; (f) $25 \% \mathrm{NH} \mathrm{OH} \mathrm{OH} \mathrm{H}_{2} \mathrm{O}$ $\mathrm{MeOH}, 60{ }^{\circ} \mathrm{C}, 3 \mathrm{~h}$

tion to the primitive bicontinuous cubic phase $\left(\mathrm{V}_{2} \mathrm{Im} 3 \mathrm{~m}\right)$ was observed at higher concentrations and only at $\mathrm{pH} 3$ due to the protonation of the phosphate headgroups.

It is proposed that the effect of the C12 phosphorylated additives on $\mathrm{MO}$ is dependent on the location of the additive within the lipid bilayer. As the $\mathrm{C} 12$ phosphorylated additives are smaller than the bulk MO lipids, they can easily move within the lipid bilayer, as dictated by their amphiphilic properties. Thus, it is hypothesised that the headgroup of the C12P1 remains below the lipid-water interface; the $\mathrm{C} 12 \mathrm{P} 3$ headgroup above the interface, and $\mathrm{C} 12 \mathrm{P} 2$ headgroup at the interface. Positioning of the phosphorylated additives is thus further investigated through the determination of its $\mathrm{pK}_{\mathrm{a}}$ in the following section.

Due to the hydrophobic mismatch between the tails of the phytol additives and the bulk MO, there is a substantial difference between the phase behaviour of the di- and tri- phosphorylated phytol surfactants (Fig. 2D-E). At concentrations as low as $0.5 \mathrm{~mol} \%$ of PhyP2, MO cannot form the cubic phase due to the considerable influence of the additive on the hydrophobic portion of the mesophase. PhyP3 on the other hand, slightly swells the lattice parameter of the $\mathrm{V}_{2}$ Pn3m (Fig. SI5) such that formation of $\mathrm{V}_{2} \mathrm{Im} 3 \mathrm{~m}$ and vesicles are observed at $2.5 \mathrm{~mol} \%$. In the case of the phytol substituted phosphorylated additives, it is hypothesised that PhyP2 remains hidden below the lipid-water interface, while the PhyP3 is above the interface. As can be seen, the mismatch between the tails in terms of length and substitution influences the positioning of the phosphorylated additives within the mesophase.

3.2.1.2. Phase behaviour of phytantriol (PHYT) $V_{2}$ Pnзm bulk phase with phytol phosphorylated lipids. The phosphorylated phytol additives were introduced into a PHYT bulk phase to investigate the effect of matching the hydrophobic portion of the additive to the bulk lipid (Fig. 2G-I). Because the length and substitutions of the lipidic tail were identical to those of PHYT, the additives were expected to be arranged such that the phosphate headgroups are aligned at the bilayer-water interface. The effect of both PhyP2 and PhyP3 on the lattice parameter (Fig. SI5 and SI6) of the PHYT $V_{2}$ Pn3m are similar to the effect of the $\mathrm{C} 12$ additives in $\mathrm{MO}$; there is a swelling of the unit cells at low concentrations, and the effect is greatest at $\mathrm{pH}$ $3>8>6$. The transition to $\mathrm{V}_{2} \operatorname{Im} 3 \mathrm{~m}$ was only observed at $\mathrm{pH} 3$ at 

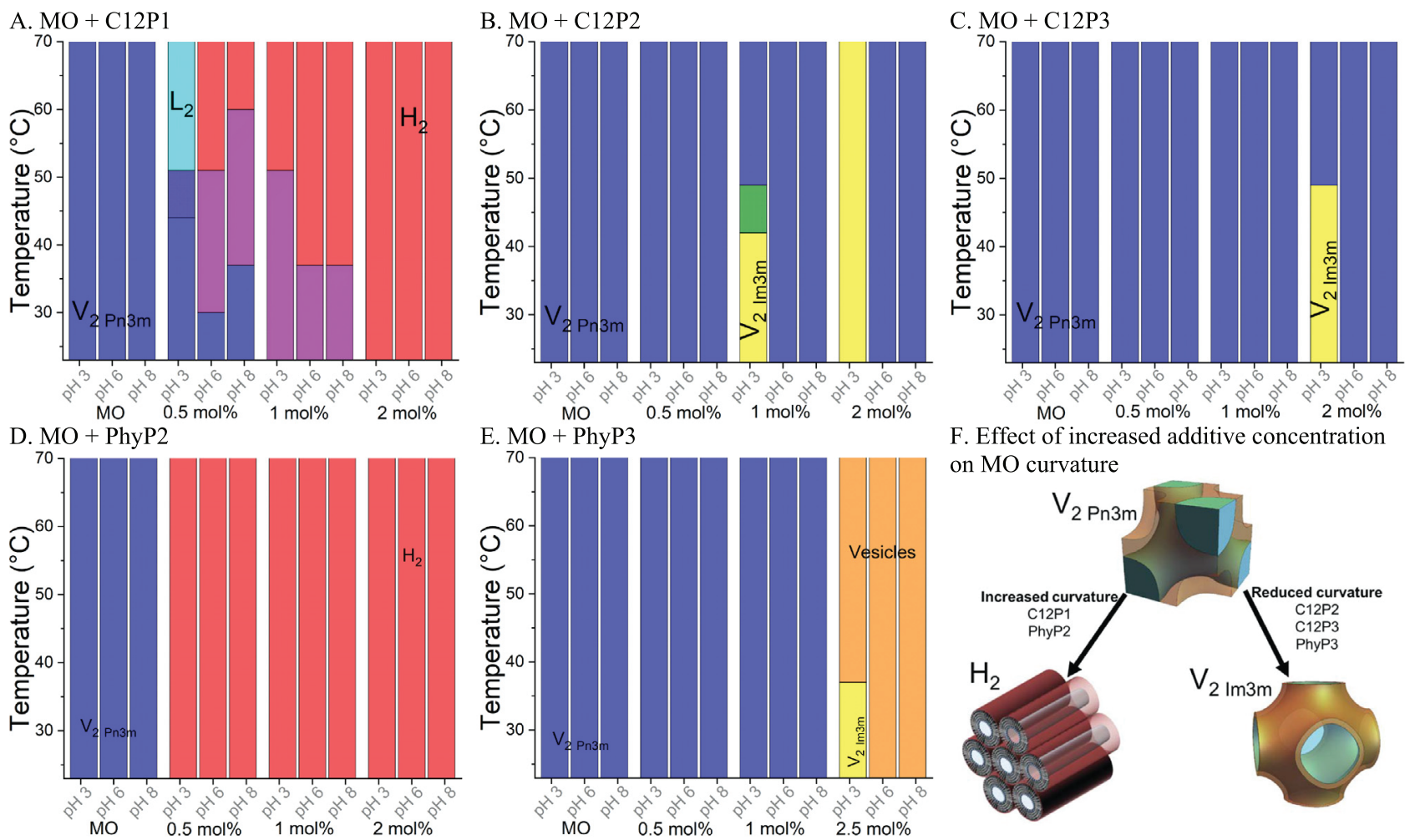

F. Effect of increased additive concentration on MO curvature
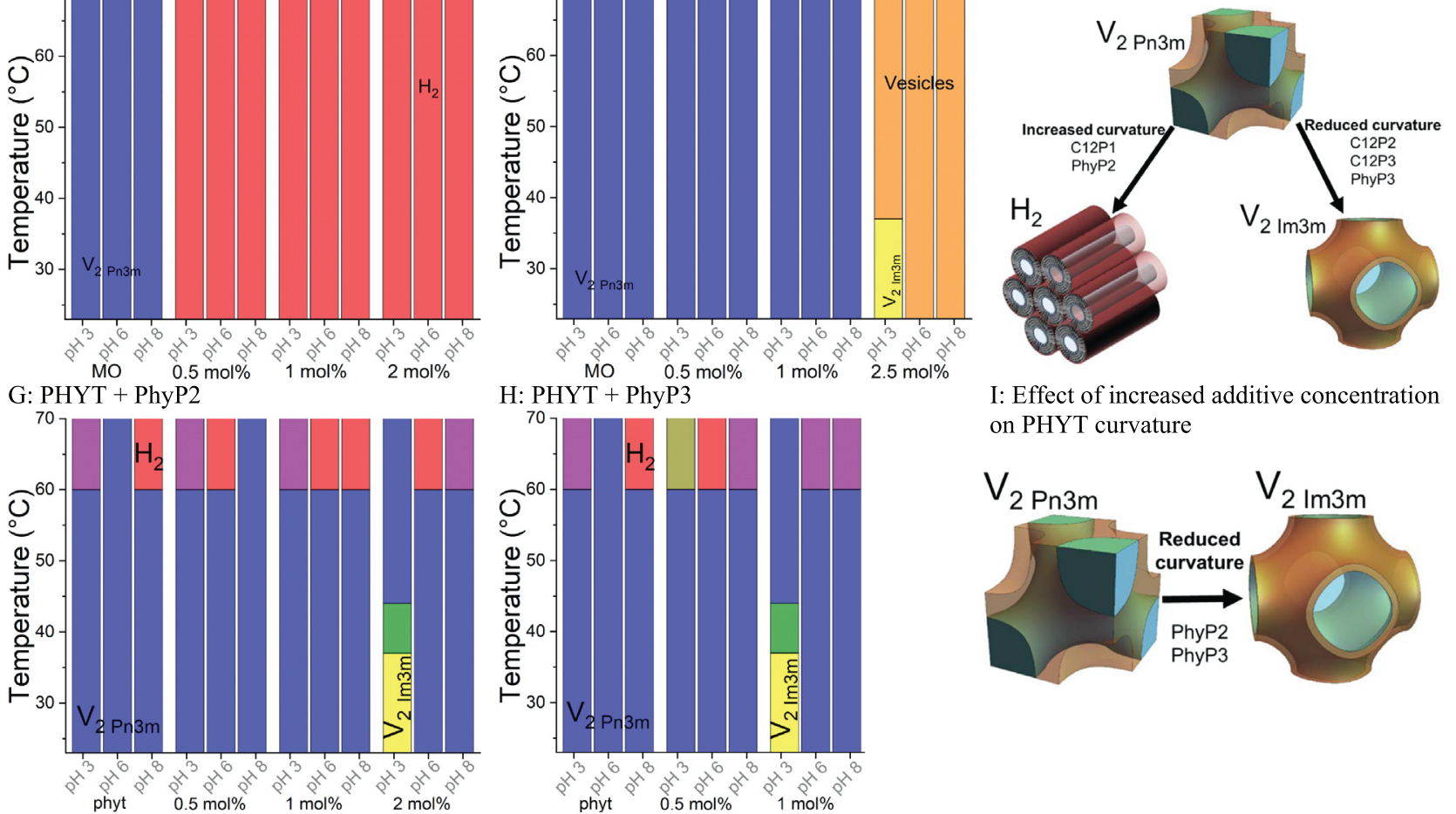

I: Effect of increased additive concentration on PHYT curvature

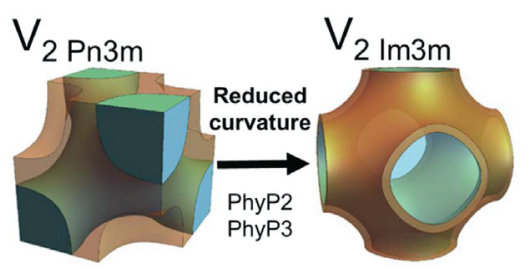

Fig. 2. Composition, temperature and $\mathrm{pH}$ - dependent phase behaviour of the phosphorylated lipid additives in monoolein bulk phase formulations in excess water as determined by SAXS. Blue: $V_{2}$ Pn3m; navy: $V_{2}$ Pn3m $+L_{2}$; cyan: $L_{2}$; purple: $V_{2}$ Pn3m $+H_{2}$; red: $H_{2}$; yellow: $V_{2}$ Im3m; green: $V_{2}$ Im3m $+V_{2}$ Pn3m; orange: vesicles and dark yellow: $V_{2}$ $\mathrm{Pn} 3 \mathrm{~m}+\mathrm{V}_{2 \text { Ia3d }}+\mathrm{H}_{2}$. Changes in lattice parameter are reported in Fig. SI1-7. A: Increasing amounts of $\mathrm{C} 12 \mathrm{P} 1$ additives results in the formation of inverse hexagonal phase $\left(\mathrm{H}_{2}\right)$ at lower temperatures at various $\mathrm{pH}$ values. B: Increasing amounts of $\mathrm{C} 12 \mathrm{P} 2$ additives results in the swelling of the unit cells, followed by the formation of $\mathrm{V}_{2}$ Im3m upon at $<1 \mathrm{~mol} \%$ at pH 3 only. C: Increasing amounts of C12P3 additives results in swelling of the unit cells, followed by formation of $\mathrm{V}_{2}$ Im3m at 2 mol\% at pH 3 only. D: Increasing amounts of PhyP2 additive results in formation of $\mathrm{H}_{2}$ from $0.5 \mathrm{~mol} \%$, and at all pH values. E: Increasing amounts of the PhyP3 additive results in swelling of the unit cells. No phase transitions were observed until $2.5 \mathrm{~mol} \%$ where formation of $\mathrm{V}_{2} \mathrm{Im} 3 \mathrm{~m}$ and of vesicles was observed. F: Schematic drawing showing the effect of increased additive concentration on MO bilayer curvature. The hydrophilic and hydrophobic regions of the $\mathrm{V}_{2}$ phase are denoted by blue and yellow respectively. The hydrophilic and hydrophobic regions of the $\mathrm{H}_{2}$ phase are denoted by blue and red respectively. G: Increasing amounts of PhyP2 additive results in swelling of the unit cells, followed by the formation of $\mathrm{V}_{2} \mathrm{Im} 3 \mathrm{~m}$ at $2 \mathrm{~mol} \%$ and $\mathrm{pH} 3$ only. $\mathrm{H}$ : Increasing amounts of the PhyP3 additive results in swelling of the unit cells, followed by formation of $\mathrm{V}_{2}$ Im3m at $1 \mathrm{~mol} \%$ and pH 3 only. Note that PhyP3 could not be incorporated into phytantriol at 2 mol\% due to its limited miscibility with the lipid. I: Schematic drawing showing the effect of increased additive concentration on PHYT lipid bilayer curvature. Schematics are . reproduced with permission from [30,31]

2 mol\% PhyP2 and at 1 mol\% PhyP3. The unusual observation of $\mathrm{V}_{2}$ Ia3d at high temperatures at $0.5 \mathrm{~mol} \% \mathrm{PhyP3}$ and at $\mathrm{pH} 3$ further demonstrates the influence of protonation over the bilayer curvature.

\subsubsection{The effect of phosphorylated lipids on cubosomes}

The pH-dependent effect of phosphorylated additives on dispersed bicontinuous cubic phases (cubosomes) was subsequently investigated (temperature dependent phase behaviour is reported in Fig. SI8 and raw SAXS data in Fig. SI9). The differences in phase behaviour between the cubosomes with and without added phosphorylated lipids were used an indication of their effect on the lipid-water interface. The guest phosphorylated lipids were added at a ratio of $0.5 \mathrm{~mol} \%$ to the main host lipid, as this was determined to be the concentration at which cubic phase architecture could be maintained across all MLO and PHYT formulations. Sharp ${ }^{31} \mathrm{P}$ NMR signals could be obtained with dispersed lipid particles, while this could not be achieved in preliminary experiments with bulk LCPs. The ${ }^{31} \mathrm{P}$ NMR spectra were used to determine the $\mathrm{pK}_{\mathrm{a}}$ of the phosphorylated molecules in solution and incorporated into cubosomes, which, in turn, was utilised as an indirect method of determining the location of the additive at the hydrophobichydrophilic interface of MLO $V_{2}$ Im3m and PHYT $V_{2}$ Pn3m cubosomes, respectively. 
It is noted that the $\mathrm{pH}$ range of MLO cubosomes was restricted to 2-10 due to susceptibility of the host monoacylglycerol to hydrolysis; glycerol and linoleic acid, the two cleavage products were found at $\mathrm{pH}$ values outside this range. Additionally, no phosphorylated additive was found in the supernatant of the lipid dispersions, thus, manipulating the hydrophilicity of the phosphorylated molecules did not lead to an accumulation of free additive in the aqueous phase. The phase behaviour of the monoacylglycerol-based cubosomes was different from the bulk phase behaviour due to addition of the polymeric stabiliser, Pluronic F127 [32,33]. Particle size of the samples are shown in Table 1.

3.2.2.1. Phase behaviour of MLO $V_{2} \operatorname{Im} 3 m$ cubosomes with C12 phosphorylated lipids. The mono- and di-phosphorylated lipids (C12P1 and $\mathrm{C} 12 \mathrm{P} 2$ ) swell the lattice parameters of the MLO $\mathrm{V}_{2} \mathrm{Im} 3 \mathrm{~m}$ cubosomes more than the tri-phosphorylated lipid, C12P3 (Fig. 3A). The presence of lamellar structures was observed in C12P1, C12P2 and $\mathrm{C} 12 \mathrm{P} 3$ dispersions at $\mathrm{pH} 5-9$, where the phosphate groups are more likely ionised. Lamellar phase was also observed at $\mathrm{pH} 2.5$ in the $\mathrm{C} 12 \mathrm{P} 3$ dispersion, where it is hypothesised that protonation of the headgroup increases the hydrophobicity of the additive, forcing the additive into the bilayer at the interface. The measured phase behaviour supports the hypothesis that the C12P1 and C12P2 additives are located at the interface, whereas the relative hydrophilicity of the $\mathrm{C} 12 \mathrm{P} 3$ draws its headgroup further into the confined water layer.

3.2.2.2. Phase behaviour of MLO $V_{2}$ Im3m cubosomes with phytol-based phosphorylated lipids (Fig. 3B). The $\mathrm{pH}$ dependent phase behaviour of cubosomes doped with PhyP2 reveal coexistence with lamellar phases at low $\mathrm{pH}$. In this case, protonation of the PhyP2 headgroups presumably affects a reduction in membrane curvature due to charge neutralisation and consequent insertion of the lipid into the interface. Little difference could be observed between formulations with PhyP3 and pure MLO cubosomes over the measured $\mathrm{pH}$ range.

3.2.2.3. Phase behaviour of phytantriol $V_{2}$ Pn3m $c u b o s o m e s$ with phytolbased phosphorylated lipids. At basic $\mathrm{pH}$, the ionisation and the resulting hydration of the headgroups of both PhyP2 and PhyP3 results in the formation of $\mathrm{V}_{2} \mathrm{Im} 3 \mathrm{~m}$ at $\mathrm{pH} 8.5$ and 7.1 respectively (Fig. 3C). Thus, in contrast to the observed behaviour of the bulk phase, where $\mathrm{V}_{2} \operatorname{Im} 3 \mathrm{~m}$ only occurred at low $\mathrm{pH}$, here ionisation results in a partial order-order phase transition to $\mathrm{V}_{2} \mathrm{Im} 3 \mathrm{~m}$. This is attributed primarily to the additional degrees of freedom that have been introduced into the system by addition of the polymeric stabiliser, Pluronic F127 [32,33], and secondly the increase in surface area and curvature of the particles compared to the bulk [34].

\subsection{3. $p K_{a}$ determination of phosphorylated lipids in cubosomes}

The $\mathrm{pK}_{\mathrm{a}}$ of a molecule at the lipid-water interface is determined by the interplay between the energetic costs for burying a charged moiety into the apolar bilayer and the hydrophobic interactions between the free and lipid-bound fatty acid chains [35]. Additionally, H-bonds can form between charged and protonated forms, where the formed H-bonds stabilise the charged form at the water-oil interface [35]. Thus, the extent of the $\mathrm{pK}_{\mathrm{a}}$ shift between micellar solution and cubosomes was used as an indirect method to determine the positioning of the additive in the lipid bilayer.

${ }^{31} \mathrm{P}$ NMR spectroscopy was used in an innovative way in order to establish $\mathrm{pK}_{\mathrm{a}}$ titration curves. Diffusion NMR and solid-state NMR spectroscopy have previously been utilised to identify the movement and location of additives in bulk lipid bilayers [21,3638]. However, instead of using these advanced NMR techniques which directly measure the diffusion of molecules in the mesophase, solution state ${ }^{31} \mathrm{P}$ NMR was utilised to detect chemical shifts
Table 1

Particle size of the as-made lipid particles determined by dynamic light scattering.

\begin{tabular}{lll}
\hline Sample name & z-ave (d.nm) & PDI \\
\hline MLO & 196.9 & 0.147 \\
MLO + 0.5C12P1 & 121.1 & 0.114 \\
MLO + 0.5C12P2 & 190.2 & 0.255 \\
MLO + 0.5C12P3 & 203.7 & 0.259 \\
MLO + 0.5phyP2 & 187.5 & 0.233 \\
MLO + 0.5phyP3 & 181.3 & 0.124 \\
phyt & 238.4 & 0.239 \\
phyt + 0.5phyP2 & 257.4 & 0.279 \\
phyt + 0.5phyP3 & 237.5 & 0.292 \\
\hline
\end{tabular}

of a phosphate group, which are dependent on the degree of protonation [39-41]. Using these chemical shifts, $\mathrm{pK}_{\mathrm{a}}$ titration curves were constructed. Although disruptive effects of salts upon ${ }^{31} \mathrm{P}$ NMR measurements have been reported, for example $\mathrm{Mg}^{2+}$ and $\mathrm{K}^{+}$in biological probes [42], problems were not encountered in investigating these self-assembled matrices as concentration and other conditions were kept constant within the series of measurements. The measured $\mathrm{pK}_{\mathrm{a}}$ values of each phosphate of the lipid headgroup are in good agreement with those predicted by WESTHEIMER, where $\mathrm{pK}_{\mathrm{a}(1)}$ and $\mathrm{pK}_{\mathrm{a}(2)}$ are approximately 2 and 7, respectively [43]. In this study, the focus is upon a shift in $\mathrm{pK}_{\mathrm{a}(2)}$ of the phosphates on each headgroup as it occurs at a more accessible and physiologically relevant $\mathrm{pH}$, and avoids the extreme $\mathrm{pH}$ values at which hydrolysis of the main host lipid can occur.

3.2.3.1. MLO cubosomes + phosphorylated lipids. The $\mathrm{pK}_{\mathrm{a}}$ values of the lipid additives in micellar solution and MLO cubosomes are tabulated in Table 2. Titration curves and molecular annotations are reported in Fig. SI9-11, where phosphate groups are labelled starting from primary phosphoester linkage (1) moving along the molecule increasing the number for the secondary or tertiary group (2), (3)).

Clear shifts in $\mathrm{pK}_{\mathrm{a} 2}$ values of almost all phosphate groups were observed when comparing the micellar solution and the MLO cubosomes, demonstrating an altered environment for the phosphates in the headgroups. For both the $\mathrm{C} 12$ and phytol phosphorylated lipids, the overall observed change of each $\mathrm{pK}_{\mathrm{a}}$ is dependent on the number of phosphates on the headgroup. The shift in $\mathrm{pK}_{\mathrm{a} 2}$ of the diphosphates (C12P2 and PhyP2) was found to be two to three times greater than that observed for the triphosphates (C12P3 and PhyP3). This larger shift demonstrates that the diphosphates experience a larger protection from deprotonation as they are preferentially arranged at the interface where they exert more influence on the lipid packing. This agrees with the changes in bulk phase behaviour upon changes in $\mathrm{pH}$; the triphosphorylated lipids protrude out of the interface into the confined aqueous layer as they are more hydrophilic, where they promote the increased hydration of the lipid bilayer and consequently the formation of $V_{2} \operatorname{Im} 3 \mathrm{~m}$ or lamellar phase.

The magnitude of shift to higher $\mathrm{pK}_{\mathrm{a}}$ values was observed to be in the order of PhyP2 $>\mathrm{C} 12 \mathrm{P} 2>\mathrm{C} 12 \mathrm{P} 3$, where a larger shift was observed for (2) and (3) than for (1). This is due to a larger difference in the environment of the individual phosphate groups. The primary phosphate, (1), exists in an environment in which it is closer to its state when in micellar solution. Whereas the larger change in $\mathrm{pK}_{\mathrm{a}}$ of (2) and (3) indicates their interactions with the headgroups of other surfactants at the lipid-water interface. In comparison, the shift in $\mathrm{pK}_{\mathrm{a} 2}$ of the phosphate groups in PhyP3 demonstrates a different behaviour as both positive and negative shifts were observed. The primary phosphate, (1), exhibits a positive shift, whereas the (2) a negative shift in $\mathrm{pK}_{\mathrm{a}}$. No change was observed for (3). This unexpected behaviour could be caused by a hydropho- 

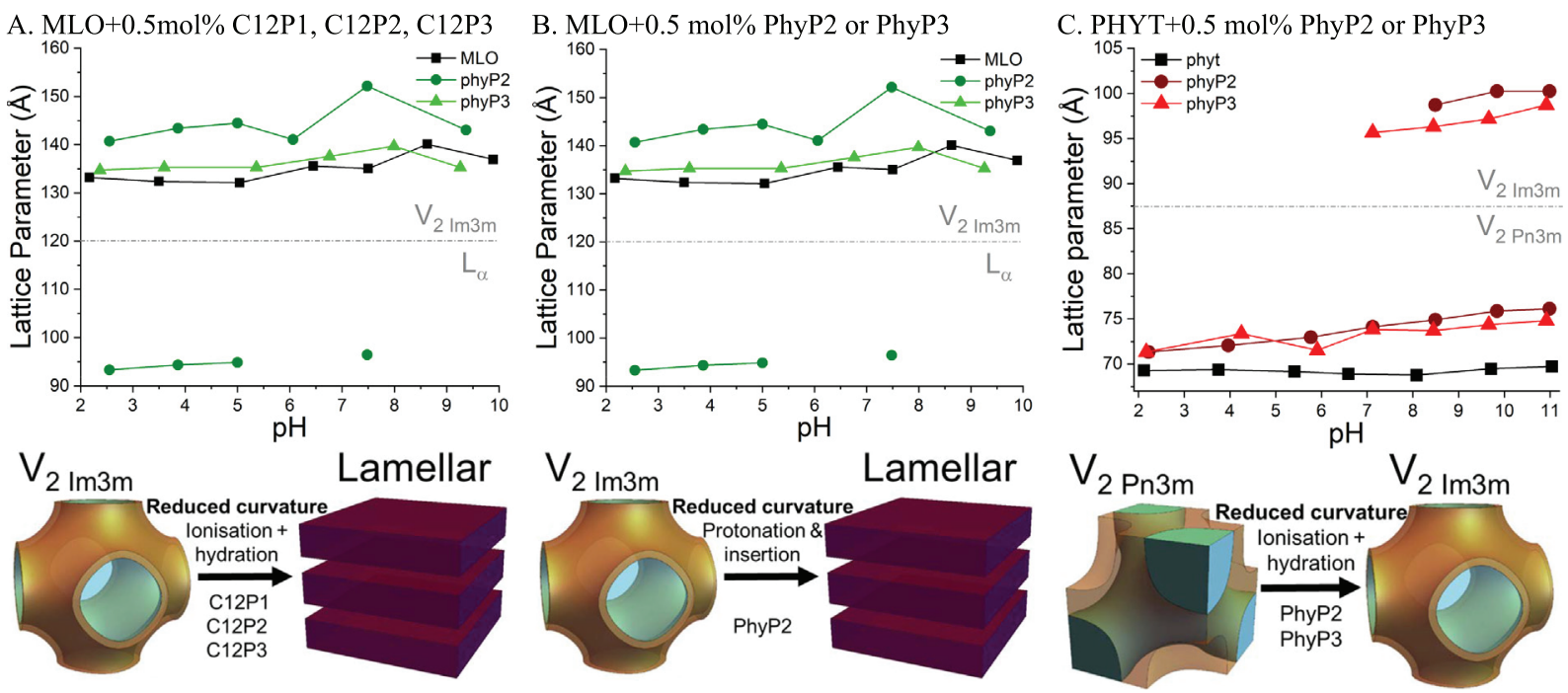

Fig. 3. $\mathrm{pH}$ dependent phase behaviour of host-guest cubosomes as determined by SAXS at $23{ }^{\circ} \mathrm{C}$. The phase boundaries are indicated by dotted lines and annotated accordingly. All formulations contained $0.5 \%$ of phosphorylated lipid as additive. The temperature dependent phase behaviour is reported in Fig. SI8. Each panel contains a schematic drawing demonstrating the effect of increased additive concentration on lipid bilayer curvature. The hydrophilic and hydrophobic regions of the $\mathrm{V}_{2}$ phase are denoted by blue and yellow respectively. The hydrophilic and hydrophobic regions of the $\mathrm{H}_{2}$ phase are denoted by blue and red respectively. Schematics are . reproduced with permission from $[21,31]$

Table 2

$\mathrm{pK}_{\mathrm{a} 2}$ values of the phosphate headgroups of phosphorylated additives in micellar solution (left three columns), upon incorporation into MLO-based cubosomes (central three columns) and their difference (right three columns).

\begin{tabular}{|c|c|c|c|c|c|c|c|c|c|}
\hline & \multicolumn{3}{|c|}{ Micellar solution } & \multicolumn{3}{|c|}{ MLO-based cubosomes } & \multicolumn{3}{|c|}{ Difference } \\
\hline & $\mathrm{pK}_{\mathrm{a} 2(1)}$ & $\mathrm{pK}_{\mathrm{a} 2(2)}$ & $\mathrm{pK}_{\mathrm{a} 2(3)}$ & $\mathrm{pK}_{\mathrm{a} 2(1)}$ & $\mathrm{pK}_{\mathrm{a} 2(2)}$ & $\mathrm{pK}_{\mathrm{a} 2(3)}$ & $\mathrm{pK}_{\mathrm{a} 2(1)}$ & $\mathrm{pK}_{\mathrm{a} 2(2)}$ & $\mathrm{pK}_{\mathrm{a} 2}(3)$ \\
\hline C12P1 (3) & - & - & - & $8.55 \pm 0.15$ & - & - & - & - & - \\
\hline $\mathrm{C} 12 \mathrm{P} 2(\mathbf{6})$ & $6.89 \pm 0.06$ & $7.62 \pm 0.04$ & - & $7.62 \pm 0.11$ & $8.51 \pm 0.10$ & - & 0.79 & 0.89 & - \\
\hline C12P3 (13) & $7.23 \pm 0.07$ & $7.69 \pm 0.06$ & $8.41 \pm 0.04$ & $7.33 \pm 0.11$ & $7.94 \pm 0.08$ & $8.62 \pm 0.11$ & 0.10 & 0.25 & 0.21 \\
\hline PhyP2 (17) & $6.97 \pm 0.03$ & $7.94 \pm 0.02$ & - & $7.79 \pm 0.16$ & $9.32 \pm 0.43$ & - & 0.82 & 1.38 & - \\
\hline PhyP3 (9) & $6.86 \pm 0.01$ & $8.20 \pm 0.06$ & $8.80 \pm 0.04$ & $7.34 \pm 0.01$ & $7.93 \pm 0.11$ & $8.79 \pm 0.20$ & 0.48 & -0.27 & -0.01 \\
\hline
\end{tabular}

bic mismatch between the bulk MLO and the phytol chain where the incompatibility in chain length can increase intermolecular distance, leading to a decrease in apparent $\mathrm{pK}_{\mathrm{a}}[44,45]$.

3.2.3.2. Phytantriol-based cubosomes. The overall shift in $\mathrm{pK}_{\mathrm{a} 2}$ values of both PhyP2 and PhyP3 in PHYT-based cubosomes was greater than that of the MLO-based cubosomes. This is attributed to matching of the phytol tails of the guest and host PHYT lipid (Fig. SI12 and Table 3). The shifts in $\mathrm{pK}_{\mathrm{a} 2}$ values of (1) and (2) in PhyP2 were almost identical, indicating that both phosphate groups experience a similar environment at the bilayer interface. A shift in the $\mathrm{pK}_{\mathrm{a}}$ of the phosphorylated lipid additives in PHYT cubosomes was observed in all three phosphates on the PhyP3 headgroup, where the positive shift in $\mathrm{pK}_{\mathrm{a}}$ was greatest for the primary (1) > (3) > (2)phosphate groups. These values support the phase transitions observed with increasing $\mathrm{pH}$, as the deprotonation and subsequent hydration of the phosphate headgroups occur at the bilayer interface.

\section{Discussion}

\subsection{Location is the key to influence lipid packing}

The position of an amphiphilic guest additive in the host bilayer influences bilayer curvature. In this study, it was determined that the headgroup of the surfactant positions itself below, at or above the lipid-water interface, as determined by the balance of hydrophilic and hydrophobic properties of the molecules.

The concentration and $\mathrm{pH}$ dependent phase behaviour of the C12 phosphorylated lipids afforded insight into the preferred location of the phosphate groups at the interface. As the chains of the guest C12 phosphorylated lipids are shorter than those of the host monoacylglycerols, the molecules acquire a degree of mobility that was dictated by the size and protonation state of the phosphorylated headgroup. C12P1 is more likely to reside in the hydrophobic region, thus promoting the formation of mesophases with increasing negative curvature [14]. C12P3, the most hydrophilic additive tested, appeared to be located with its headgroup protruding out of the bilayer into the aqueous domain. C12P2 has a more ideal hydrophilic-hydrophobic balance and is thus positioned at the interface such that it can exert maximal influence upon lipid packing.

The hydrophobic mismatch and acyl-chain stretching of amphiphilic molecules in membranes can result in phase separation of lipids, ordering and disordering of the acyl chains, local deformation or phase transitions, albeit little is known about which is favoured under certain conditions $[46,47]$. The hydrophobic mismatch between the phytol-containing guest additives and the host monoacylglycerols appears to have resulted in the phosphorylated additives assembling either with their headgroups below the interface, or entirely outside of the lipid bilayer regardless of the $\mathrm{pH}$, as they have less degrees of freedom. When the tails of the phosphorylated lipids match those of the phytantriol mesophases, the phosphorylated additives appear to follow the same trend in head- 
Table 3

$\mathrm{pK}_{\mathrm{a} 2}$ values of the phosphate headgroups of the phosphorylated additives in micellar solution (left three columns), upon incorporation into PHYT-based cubosomes (central three columns) and their difference (right three columns).

\begin{tabular}{|c|c|c|c|c|c|c|c|c|c|}
\hline & \multicolumn{3}{|c|}{ Micellar solution } & \multicolumn{4}{|c|}{ PHYT Cubosomes } & \multicolumn{2}{|c|}{ Difference } \\
\hline & $\mathrm{pK}_{\mathrm{a} 2(1)}$ & $\mathrm{pK}_{\mathrm{a} 2(2)}$ & $\mathrm{pK}_{\mathrm{a} 2(3)}$ & $\mathrm{pK}_{\mathrm{a} 2(1)}$ & $\mathrm{pK}_{\mathrm{a} 2(2)}$ & $\mathrm{pK}_{\mathrm{a} 2(2)}$ & $\mathrm{pK}_{\mathrm{a} 2(1)}$ & $\mathrm{pK}_{\mathrm{a} 2(2)}$ & $\mathrm{pK}_{\mathrm{a} 2(3)}$ \\
\hline PhyP2 (17) & $6.97 \pm 0.03$ & $7.94 \pm 0.02$ & - & $8.89 \pm 0.05$ & $9.88 \pm 0.13$ & - & 1.92 & 1.94 & - \\
\hline PhyP3 (9) & $6.86 \pm 0.01$ & $8.20 \pm 0.06$ & $8.80 \pm 0.04$ & $7.80 \pm 0.11$ & $8.88 \pm 0.18$ & $9.54 \pm 0.27$ & 0.94 & 0.68 & 0.74 \\
\hline
\end{tabular}

group position as the $\mathrm{C} 12$ additives. Of all the systems investigated in this study, the diphosphorylated additives show an ideal positioning in the lipid bilayer, provided there is no mismatch in the hydrophobic groups. As can be seen, the size of the headgroups constitutes the main influence on lipid packing, while it is the tail group that determines its ultimate position.

\subsection{Ionisation vs protonation}

The balance between hydrophobic and hydrophilic forces is easily manipulated via changes in $\mathrm{pH}$. At $\mathrm{pH}<\mathrm{pK}_{\mathrm{a}}$, protonation appears to increase hydrophobicity of the phosphorylated lipidic acids, which can result in two effects: firstly, an increase the formation of hydrogen bonds with neighbouring headgroups or water molecules [48]; and secondly, desolvation of the ionisable group that forces the neutral species into the interface and further into the bilayer. At $\mathrm{pH} \sim \mathrm{pK}_{\mathrm{a}}$, a partial deprotonation step takes place resulting in the decreased likelihood of the phosphate headgroup to form H-bonds, which leads to a smaller overall headgroup size. At $\mathrm{pH}>\mathrm{pK}_{\mathrm{a}}$, the phosphate groups are more likely to be fully deprotonated, thereby increasing the charge density at the phosphate headgroup and increasing hydration. In turn, this can lead to two outcomes: swelling of the unit cells, as seen with the diphosphorylated additives, and/or protrusion of the headgroup of the guest molecule from the interface into the aqueous layer where it is less able to influence the curvature of the lipid bilayer, as was observed with the triphosphorylated additives [49].

\section{Conclusions}

Previously reported studies have demonstrated that incorporation of amphiphilic additives can swell the lipidic cubic phase by influencing the overall curvature of the lipid bilayer $[2,9,13,15]$. This study demonstrates the effect of headgroup size and charge upon bulk and dispersed lipidic cubic phases. A library of mono-, di- and triphosphorylated lipidic molecules was designed, synthesised and incorporated into monoglyceride- and phytantriol-based mesophases, and the ensuing phase behaviour was determined by SAXS. The $\mathrm{pK}_{\mathrm{a}(2)}$ of the phosphorylated lipids at the interface was determined by ${ }^{31} \mathrm{P}$ NMR as a unique, label-free indication of their positioning at the lipid-water interface. The positioning of the additives at the interface was found to be the main determinant of their influence on lipid curvature. In agreement with literature, the position of the additive at the lipid-water interface is mostly determined by the compatibility of its hydrophobic portion with the bulk lipid $[46,47]$. Most importantly, however, is the location of the headgroup relative to the interface which was manipulated by altering the number of phosphates substituted into the headgroup and their ionisation state. In bulk mesophases, the influence of protonation was found to be greater than ionisation, whereas in dispersed phases, the opposite was observed; ionisation had a greater effect upon lipid curvature than protonation. The presented results constitute a considerable step towards elucidating the factors that influence the packing of amphiphilic molecules at interfaces, thus directing the formulation of lipidic cubic phases for application in various fields such as catalysis, drug delivery and membrane protein crystallisation or reconstitution.

\section{CRediT authorship contribution statement}

Marco Etter: Conceptualization, Investigation, Formal analysis, Writing - original draft. Daria Dellenbach: Investigation. Alke Petri-Fink: Supervision, Resources, Writing - review \& editing. Barbara Rothen-Rutishauser: Supervision, Resources, Writing review \& editing. Ehud M. Landau: Project administration, Supervision, Writing - review \& editing. Wye-Khay Fong: Conceptualization, Resources, Project administration, Investigation, Formal analysis, Writing - original draft.

\section{Declaration of Competing Interest}

The authors declare that they have no known competing financial interests or personal relationships that could have appeared to influence the work reported in this paper.

\section{Acknowledgements}

This work was supported by the Swiss National Science Foundation through the National Center of Competence in Research BioInspired Materials and the Adolphe Merkle Foundation.

\section{Appendix A. Supplementary material}

Supplementary data to this article can be found online at https://doi.org/10.1016/j.jcis.2019.11.087.

\section{References}

[1] M. Duss, L. Salvati Manni, L. Moser, S. Handschin, R. Mezzenga, H.J. Jessen, E.M. Landau, Lipidic mesophases as novel nanoreactor scaffolds for organocatalysts: heterogeneously catalyzed asymmetric aldol reactions in confined water, ACS Appl. Mater. Interfaces 10 (5) (2018) 5114-5124.

[2] W. Sun, J.J. Vallooran, A. Zabara, R. Mezzenga, Controlling enzymatic activity and kinetics in swollen mesophases by physical nano-confinement, Nanoscale 6 (12) (2014) 6853-6859.

[3] E.M. Landau, J.P. Rosenbusch, Lipidic cubic phases: a novel concept for the crystallization of membrane proteins, Proc. Natl. Acad. Sci. USA 93 (25) (1996) $14532-14535$.

[4] E. Pebay-Peyroula, G. Rummel, J.P. Rosenbusch, E.M. Landau, X-ray structure of bacteriorhodopsin at 2.5 Angstroms from microcrystals grown in lipidic cubic phases, Science 277 (5332) (1997) 1676-1681.

[5] W.-K. Fong, R. Negrini, J.J. Vallooran, R. Mezzenga, B.J. Boyd, Responsive selfassembled nanostructured lipid systems for drug delivery and diagnostics, J. Colloid Interface Sci. 484 (2016) 320-339.

[6] R. Mezzenga, J.M. Seddon, C.J. Drummond, B.J. Boyd, G.E. Schröder-Turk, L. Sagalowicz, Nature-inspired design and application of lipidic lyotropic liquid crystals, Adv. Mater. (2019) 1900818.

[7] A.I. Tyler, H.M. Barriga, E.S. Parsons, N.L. McCarthy, O. Ces, R.V. Law, J.M. Seddon, N.J. Brooks, Electrostatic swelling of bicontinuous cubic lipid phases, Soft Matter 11 (16) (2015) 3279-3286.

[8] L. van 't Hag, S.L. Gras, C.E. Conn, C.J. Drummond, Lyotropic liquid crystal engineering moving beyond binary compositional space - ordered nanostructured amphiphile self-assembly materials by design, Chem. Soc. Rev. 46 (10) (2017) 2705-2731.

[9] B. Angelov, A. Angelova, M. Ollivon, C. Bourgaux, A. Campitelli, Diamond-type lipid cubic phase with large water channels, J. Am. Chem. Soc. 125 (24) (2003) 7188-7189. 
[10] R. Negrini, R. Mezzenga, Diffusion, molecular separation, and drug delivery from lipid mesophases with tunable water channels, Langmuir 28 (47) (2012) $16455-16462$.

[11] V. Cherezov, J. Clogston, Y. Misquitta, W. Abdel-Gawad, M. Caffrey, Membrane protein crystallization in meso: lipid type-tailoring of the cubic phase, Biophys. J. 83 (6) (2002) 3393-3407.

[12] S.J. Li, Y. Yamashita, M. Yamazaki, Effect of electrostatic interactions on phase stability of cubic phases of membranes of monoolein/dioleoylphosphatidic acid mixtures, Biophys. J. 81 (2) (2001) 983-993.

[13] J. Engblom, Y. Miezis, T. Nylander, V. Razumas, K. Larsson, On the swelling of monoolein liquid-crystalline aqueous phases in the presence of distearoylphosphatidylglycerol, Prog. Coll. Pol. Sci. S 116 (2001) 9-15.

[14] Y. Aota-Nakano, S.J. Li, M. Yamazaki, Effects of electrostatic interaction on the phase stability and structures of cubic phases of monoolein/oleic acid mixture membranes, Biochim. Biophys. Acta, Biomembr. 1461 (1) (1999) 96-102.

[15] E. Nazaruk, E. Górecka, Y.M. Osornio, E.M. Landau, R. Bilewicz, Charged additives modify drug release rates from lipidic cubic phase carriers by modulating electrostatic interactions, J. Electroanal. Chem. 819 (2018) 269274.

[16] C. Brasnett, G. Longstaff, L. Compton, A. Seddon, Effects of cations on the behaviour of lipid cubic phases, Sci. Rep. 7 (1) (2017) 8229.

[17] R.H. Templer, J.M. Seddon, P.M. Duesing, R. Winter, J. Erbes, Modeling the phase behavior of the inverse hexagonal and inverse bicontinuous cubic phases in 2:1 fatty acid/phosphatidylcholine mixtures, J. Phys. Chem. B 102 (37) (1998) 7262-7271.

[18] S. Salentinig, L. Sagalowicz, O. Glatter, Self-assembled structures and pKa value of oleic acid in systems of biological relevance, Langmuir 26 (14) (2010) 11670-11679.

[19] Y.-D. Dong, I. Larson, T. Hanley, B.J. Boyd, Bulk and dispersed aqueous phase behavior of phytantriol: effect of vitamin E acetate and F127 polymer on liquid crystal nanostructure, Langmuir 22 (23) (2006) 9512-9518.

[20] C. Fong, T. Le, C.J. Drummond, Lyotropic liquid crystal engineering-ordered nanostructured small molecule amphiphile self-assembly materials by design, Chem. Soc. Rev. 41 (3) (2012) 1297-1322.

[21] L. Salvati Manni, S. Assenza, M. Duss, J.J. Vallooran, F. Juranyi, S. Jurt, O. Zerbe, E.M. Landau, R. Mezzenga, Soft biomimetic nanoconfinement promotes amorphous water over ice, Nat. Nanotechnol. (2019).

22] O.G. Mouritsen, Lipidology and lipidomics-quo vadis? A new era for the physical chemistry of lipids, Phys. Chem. Chem. Phys. 13 (43) (2011) 1919519205.

[23] J.M. Seddon, A.M. Squires, C.E Conn, O. Ces, AJ. Heron, X Mulet, G.C. Shearman, R.H. Templer, Pressure-jump X-ray studies of liquid crystal transitions in lipids, Philos. Trans. A Math. Phys. Eng. Sci. 364 (1847) (2006) 2635-2655.

[24] W.K. Fong, T.L. Hanley, B. Thierry, A. Tilley, N. Kirby, L.J. Waddington, B.J. Boyd, Understanding the photothermal heating effect in non-lamellar liquid crystalline systems, and the design of new mixed lipid systems for photothermal on-demand drug delivery, Phys. Chem. Chem. Phys. 16 (45) (2014) 24936-24953.

[25] A. Zabara, JT.Y, Chong, I. Martiel, L. Stark, B.A. Cromer, C. Speziale, CJ. Drummond, R. Mezzenga, Design of ultra-swollen lipidic mesophases for the crystallization of membrane proteins with large extracellular domains, Nat. Commun. 9 (2018) 544.

[26] K.J. Tangso, W.-K. Fong, T. Darwish, N. Kirby, B.J. Boyd, T.L. Hanley, Nove spiropyran amphiphiles and their application as light-responsive liquid crystalline components, J. Phys. Chem. B 117 (35) (2013) 10203-10210.

27] B.L. AG, BÜCHI Vacuum Controller B-721, BÜCHI Labortechnik AG; 1996. p. 130.

[28] S.T. Hyde, Identification of lyotropic liquid crystalline mesophases. In: K. Holmberg (Ed.), Handbook of Applied Surface and Colloid Chemistry, John Wiley \& Sons, 2002.
[29] J. Loebus, B. Leitenmaier, D. Meissner, B. Braha, G.-J. Krauss, D. Dobritzsch, E. Freisinger, The major function of a metallothionein from the aquatic fungus Heliscus lugdunensis is cadmium detoxification, J. Inorg. Biochem. 127 (2013) 253-260.

[30] A. Zabara, R. Negrini, P. Baumann, O. Onaca-Fischer, R. Mezzenga, Reconstitution of OmpF membrane protein on bended lipid bilayers: perforated hexagonal mesophases, Chem. Commun. 50 (20) (2014) 2642 2645.

[31] S. Assenza, R. Mezzenga, Curvature and bottlenecks control molecular transport in inverse bicontinuous cubic phases, J. Chem. Phys. 148 (5) (2018) 054902.

[32] M. Nakano, T. Teshigawara, A. Sugita, W. Leesajakul, A. Taniguchi, T. Kamo, H. Matsuoka, T. Handa, Dispersions of Liquid crystalline phases of the monoolein/ oleic acid/pluronic F127 system, Langmuir 18 (24) (2002) 9283-9288.

[33] J.Y.T. Chong, X. Mulet, L.J. Waddington, B.J. Boyd, C.J. Drummond, Highthroughput discovery of novel steric stabilizers for cubic lyotropic liquid crystal nanoparticle dispersions, Langmuir 28 (25) (2012) 9223-9232.

[34] D. Wang, R.J. Nap, I. Lagzi, B. Kowalczyk, S. Han, B.A. Grzybowski, I. Szleifer, How and why nanoparticle's curvature regulates the apparent pKa of the coating ligands, J. Am. Chem. Soc. 133 (7) (2011) 2192-2197.

[35] M.P. Andersson, M.H.M. Olsson, S.L.S. Stipp, Predicting the pKa and stability of organic acids and bases at an oil-water interface, Langmuir 30 (22) (2014) 6437-6445.

[36] P.O. Eriksson, G. Lindblom, Lipid and water diffusion in bicontinuous cubic phases measured by NMR, Biophys. J. 64 (1) (1993) 129-136.

[37] T.G. Meikle, A. Sethi, D.W. Keizer, J.J. Babon, F. Separovic, P.R. Gooley, C.E. Conn, S. Yao, Heteronuclear NMR spectroscopy of proteins encapsulated in cubic phase lipids, J. Magn. Reson. 305 (2019) 146-151.

[38] Y. Yang, H.W. Yao, M. Hong, Distinguishing bicontinuous lipid cubic phases from isotropic membrane morphologies using P-31 solid-state NMR spectroscopy, J. Phys. Chem. B 119 (15) (2015) 4993-5001.

[39] R.F. Mortlock, A.T. Bell, C.J. Radke, Phosphorus-31 and aluminum-27 NMR investigations of the effects of $\mathrm{pH}$ on aqueous solutions containing aluminum and phosphorus, J. Phys. Chem. 97 (3) (1993) 775-782.

[40] G.K. Radda, P.J. Seeley, Recent studies on cellular metabolism by nuclear magnetic resonance, Annu. Rev. Physiol. 41(1) (1979) 749-769.

[41] R. Jayasundar, L.D. Hall, N.M. Bleehen, Comparison of pH measurements made using 31P NMR and a fibreoptic pH meter, NMR Biomed 5 (6) (1992) 360-363.

[42] J.K.M. Roberts, N. Wade-Jardetzky, O. Jardetsky, Intracellular pH measurements by phosphorus-31 nuclear magnetic resonance. influence of factors other than pH on phosphorus-31 chemical shifts, Biochemistry 20 (19) (1981) 5389-5394.

[43] F.H. Westheimer, Why nature chose phosphates, Science 235 (4793) (1987) 1173-1178.

[44] J.R. Kanicky, D.O. Shah, Effect of degree, type, and position of unsaturation on the pKa of long-chain fatty acids, J. Colloid Interface Sci. 256 (1) (2002) 201 207.

[45] J.R. Kanicky, D.O. Shah, Effect of premicellar aggregation on the pKa of fatty acid soap solutions, Langmuir 19 (6) (2003) 2034-2038.

[46] J.A. Killian, Hydrophobic mismatch between proteins and lipids in membranes, Biochimica et Biophysica Acta (BBA) - Rev. Biomembr 1376 (3) (1998) 401 416.

[47] E.J. Wallace, N.M. Hooper, P.D. Olmsted, Effect of hydrophobic mismatch on phase behavior of lipid membranes, Biophys. J. 90 (11) (2006) 4104-4118.

[48] D.L. Gater, V. Réat, G. Czaplicki, O. Saurel, F. Jolibois, V. Cherezov, A. Milon, Hydrogen bonding of cholesterol in the lipidic cubic phase, Langmuir: ACS J. Surf. Colloids 29 (25) (2013) 8031-8038.

[49] J. Kim, W. Lu, W. Qiu, L. Wang, M. Caffrey, D. Zhong, Ultrafast hydration dynamics in the lipidic cubic phase: discrete water structures in nanochannels, J. Phys. Chem. B 110 (43) (2006) 21994-22000. 